\title{
/I/IOMENTUM
}

QUARTERLY

ZEITSCHRIFT FÜR

SOZIALEN FORTSCHRITT

2020 I Vol. 9, No. 2, p. 68-84

\section{Soziale Innovation aus der Perspektive einer poststrukturalistischen Praxistheorie Kritik der parallelen Anwendung von akteur- bzw. praxistheoretischem Paradigma}

\author{
Roland Brandauer
}

Zusammenfassung

Die Dortmunder Forschungsgruppe, vertreten durch namhafte Soziolog*innen wie Howaldt, Jacobsen, Kopp und Schwarz, verfolgt das vielversprechende Projekt, Soziale Innovation durch eine (poststrukturalistische) Praxistheorie zu fundieren, um damit nicht zuletzt auch den Stellenwert der Sozialen Innovation gegenüber ökonomischer und technischer Innovation zu erhöhen. Parallel zum Paradigma einer poststrukturalistischen Praxistheorie wird dabei allerdings zugleich ein akteurtheoretisches Paradigma zum Einsatz gebracht. Diese beiden Ansätze erweisen sich jedoch in einem analytischen Theorienvergleich in Bezug auf die für eine Definition von Sozialer Innovation zentralen Begriffe von Subjekt und Intention als widersprüchlich und stark divergierend. In dieser Arbeit wird die These vertreten, dass zwar in einer multiparadigmatischen Auffassung von Wissenschaft einander widersprechende Paradigmen für eine bestimmte Zeit parallel existieren können, dass die Anwendung von sich widersprechenden Paradigmen innerhalb eines Forschungsprogramms jedoch auf logischer, inhalticher und wissenschaftstheoretischer Ebene unhaltbar ist.

Schlagwörter: Multiparadigmatische Wissenschaft, poststrukturalistische Praxistheorie, akteurtheoretisches Paradigma, Soziale Innovation

Social innovation in the perspective of a poststructuralist theory of practice: Critique of the parallel use of praxeological and actor-theoretical paradigm

\section{Abstract}

The Research Group of Dortmund, represented by well-known German sociologists like Howaldt, Jacobsen, Kopp and Schwarz, is pursuing the promising project of substantiating social innovation as a poststructuralist version of practice theory. In order to establish social innovation as a dominant 'driver' of social change towards technological and economic innovation, these scientists remain, on the other hand, faithful to the actor-theoretical paradigm of an intentional subject. In a theory comparison the two approaches are proving to be strongly divergent with regard to the central concepts of subject and intention which are of major importance in defining social innovation. This study puts forward the thesis that, although in the conception of a multi-paradigmatic science there can exist for a certain period contradictory paradigms in a parallel way, the use of contradictory paradigms within a research program is untenable at logical, content and epistemological levels.

Keywords: Multi-paradigmatic science, Poststructuralist theory of practice, Actor-theoretical paradigm, Social innovation

IIIIIIIIIIIIIIIIIIIIIIIIIIIIIIIIIIIIIIII

${ }^{*}$ Roland Brandauer. E-Mail: rolandbrandauer@yahoo.de 


\section{Einleitung}

In den Sozial- und Kulturwissenschaften besteht ein Theorienpluralismus. Als Paradebeispiel für einen multiparadigmatischen Zustand gilt die Soziologie (Gabriel et al. 2014). Seit dem Practical Turn in den Neunzigerjahren des 20. Jahrhunderts hat sich ein weiteres Paradigma herausentwickelt. Dieses praxeologische Paradigma kommt mittlerweile in zahlreichen historischen, sozial- und kulturwissenschaftlichen Gegenstandsbereichen zur Anwendung (Reckwitz 2003, 2004; Schäfer 2016, 2017).

Als paradigmatischer Rahmen bzw. als Forschungsheuristik scheint der praxistheoretische Ansatz insbesondere für die Sozial- und Kulturwissenschaft von großer Relevanz zu sein. Unter der Annahme, dass nicht Handlungen oder soziale Strukturen, sondern Praktiken die soziale Einheit bilden, können neue Perspektiven in Bezug auf soziale und kulturelle Problemfelder entwickelt werden (Reckwitz 2008a, 2008b).

Es ist daher anzunehmen, dass eine praxeologische Interpretation des Sozialen auch den Gegenstandsbereich des Sozialen Wandels und der Sozialen Innovation bzw. das Verhältnis dieser beiden zentralen Begriffe in der Soziologie und Kulturwissenschaft auf neuartige und erkenntnisgewinnende Weise $\mathrm{zu}$ beleuchten vermag.

In diesem Beitrag soll die Anwendung des praxeologischen Paradigmas (poststrukturalistische Praxistheorie) für die wissenschaftliche Untersuchung und Analyse des Gegenstandsbereichs der Sozialen Innovation reflektiert werden. Als Anwendungsbeispiel dient der Ansatz der Dortmunder Forschungsgruppe um Jürgen Howaldt, Ralf Kopp, Michael Schwarz und Heike Jacobsen. In Kapitel 3 wird das Forschungsprogramm der genannten Autor*innengruppe nachgezeichnet und interpretiert. Der soziologische Klassiker Gabriel Tarde, der wesentliche Aspekte dieses praxeologischen Ansatzes vorweggenommen hat, spielt dabei eine große Rolle. Nach Ansicht der Dortmunder Wissenschaftler*innen soll die theoretische Fundierung durch die poststrukturalistische Praxistheorie Sozialen Innovationen zu einem bedeutenderen Status gegenüber technischen und ökonomischen Innovationen verhelfen.

Hauptpunkt der vorliegenden Arbeit ist die Kritik an der parallelen Anwendung des praxeologischen Paradigmas und zusätzlich eines akteurtheoretischen Paradigmas innerhalb eines Forschungsprogramms. Auch wenn man über Thomas S. Kuhn hinausgehend die Auffassung einer multiparadigmatischen Struktur der Wissenschaften vertritt, in der einander widersprechende Paradigmen in einer Wissenschaftsdisziplin, wie z. B. der Soziologie, gleichzeitig und parallel über einen längeren Zeitraum unter Anwendung stehen, gilt das nicht für die Anwendung innerhalb eines Forschungsansatzes (Lemke/Schaal 2014; Gabriel et al. 2014). Der Gegensatz zwischen diesen beiden Paradigmen in logischer, inhaltlicher und wissenschaftstheoretischer Hinsicht wird in Kapitel 4 kritisch besprochen.

Auf inhaltlicher Ebene wird die Kritik in Kapitel 2 vorbereitet. Als Basis für die Argumentation wird das praxeologische Paradigma in Form eines analytischen Theorienvergleichs einem akteurtheoretischen Ansatz gegenübergestellt. Die Version einer poststrukturalistischen Praxistheorie von Andreas Reckwitz, auf den sich die Dortmunder Forschungsgruppe explizit bezieht, wird mit der akteurtheoretischen Soziologie von Uwe Schimank kontrastiert. Die Wahl der akteurtheoretischen Soziologie von Schimank ist darin begründet, dass es sich einerseits um eine gut ausgearbeitete Sozialtheorie handelt. Andererseits wird ein nicht ganz so „harter“ Kurs gefahren, wie es in der Rational Choice Theory der Fall ist, in der der rationale und nutzenmaximierende Akteur nach dem RPSMM-Modell charakterisiert ist: restricted, perfect informed, stable prefering, maximizing man (Lemke/Schaal 2014: 76ff.). Gemein ist diesen beiden akteurzentrierten Ansätzen das Grundmodell der erklärenden Soziologie, ${ }^{1}$ welches die Interaktion bzw. Interdependenz von Mikro- und Makroebene bzw. zwischen Akteur und sozialen Strukturen analytisch abbildet (Schimank 2016; Lemke/ Schaal 2014). Es ist also zu vermuten, dass zwischen dem Modell von Schimank und der Praxistheorie eher Anschlussmöglichkeiten bzw. Komplementaritäten bestehen als zwischen Rational Choice Theory und Praxistheorie. Der Paradigmenvergleich zeigt jedoch, dass das akteurtheoretische und das praxistheoretische Paradigma in theoretischen Kernpunkten stark divergieren, insbesondere in Bezug auf die zentralen Begriffe Subjekt und Intention (s. Kapitel 2.1 und 2.2).

Während das traditionelle Subjekt präsozial konzipiert ist und gleichsam als vorgängiger Bewusstseins-

1 Es handelt sich um das berühmte Grundmodell der Erklärenden Soziologie von James Coleman, das dann von Hartmut Esser im Sinn der Rational Choice Theory weiterentwickelt und von Schimank als Grundlage für die Konzeption einer akteurtheoretischen Soziologie verwendet wurde. Aufgrund der grafischen Darstellung gerne als „Coleman'sche Badewanne“ bezeichnet (Gabriel et al. 2014; Schimank 2016). 
block eine quasi absolute Autonomie und Souveränität aufweist, erweist sich das dekonstruierte Subjekt als kontingent und geschichtsabhängig.

Es handelt sich bei Letzterem um eine performative Konstituierung des Subjekts, die einer permanenten Wiederholung bedarf. Wobei der Witz darin besteht, dass diesen Wiederholungen und iterativen Prozessen ein Innovationspotenzial immanent ist. Denn es gibt keine perfekte Wiederholung, jede Wiederholung weicht mehr oder weniger vom Vorzustand ab. Es gibt kein Original. Es gibt keinen Anfang und kein Ende. In der Iterabilität der Subjektkonstituierung steckt ein unentwegtes Innovations- bzw. Destabilisierungspotenzial.

„[D]ie Proklamation des ,Todes des Subjekts, wie man sie bei Michel Foucault findet" (Reckwitz 2016: 69), der von Foucault postulierte Tod des Subjekts, zum Ausdruck gebracht in seiner mittlerweile schon klassischen Metapher, dass „der Mensch verschwindet wie am Meeresufer ein Gesicht im Sand“ (Foucault, 1974: 462), ist abgesagt, es herrscht im Gegenteil eine rege Forschungstätigkeit bezüglich des Subjekts, richtiger gesagt bezüglich der Prozesse der Subjektivation - nach poststrukturalistischer Praxistheorie existiert kein Subjekt ohne Subjektivation (Reckwitz 2008a; Schäfer 2016; Alkemeyer et al. 2015; Alkemeyer/Bröckling 2018).

Wie in Kapitel 5 als Ausblick und Forschungsperspektive kurz erörtert wird, weist die poststrukturalistische Praxistheorie eher eine Affinität zu neuen System- bzw. Komplexitätstheorien auf als zu akteurzentrierten Theorien.

\section{Paradigmenvergleich: akteurtheoretisches \\ Paradigma $(\mathrm{P} 1)$ versus praxistheoretisches \\ Paradigma (P2)}

In diesem vorbereitenden Teil der Arbeit geht es um die inhaltliche Begründung der Kritik am Dortmunder Forschungsprogramm. Es soll anhand eines theoretischen Vergleichs demonstriert werden, dass die gleichzeitige Verwendung von akteurtheoretischem und praxeologischem Ansatz inkompatibel ist. Im anschließenden Kapitel 3 wird dieses Forschungsprogramm näher besprochen und die parallele Anwendung der beiden Paradigmen nachgewiesen.

Als Vertreter eines akteurtheoretischen Paradigmas (P1) wird die akteurtheoretische Soziologie von Uwe Schimank gewählt, als Repräsentant des poststrukturalistisch praxeologischen Paradigmas ( $\left.\mathrm{P}_{2}\right)$ fungiert der kulturtheoretische Ansatz von Andreas Reckwitz.

Es gibt eine Vielfalt an Sozialtheorien. Ich gehe davon aus, dass Begriffe wie Subjekt und Intention nur vor dem Hintergrund der jeweils verwendeten Sozialtheorie $\mathrm{zu}$ verstehen sind. Insbesondere gehe ich davon aus, dass die Bedeutung des Begriffs Intention von den entsprechenden Subjektkonzeptionen dieser Sozialtheorien abhängig ist. Die Bedeutung eines Begriffs ist nur relativ auf andere Aussagen und Begriffe in einem ganzen System von Aussagen und Überzeugungen $\mathrm{zu}$ verstehen. Die Bedeutung eines Begriffs ist im Sinne eines semantischen Holismus eine Eigenschaft des ganzen Systems; die einzelnen Teilaussagen und Begriffe sind daher nur vom Ganzen her zu verstehen und nur von diesem ableitbar (Esfeld 2002). Im folgenden Abschnitt soll die unterschiedliche Verwendungsweise der für die Analyse von Sozialer Innovation zentralen Begriffe Subjekt und Intention in der akteurtheoretischen Soziologie Schimanks bzw. in der poststrukturalistischen Praxistheorie von Reckwitz verglichen werden.

Das sozialwissenschaftliche Projekt von Schimank besteht darin, funktionale Differenzierung akteurtheoretisch zu erklären bzw. - anders ausgedrückt - das systemtheoretische Konzept in kritischer Auseinandersetzung mit Luhmanns Systemtheorie akteurtheoretisch zu rekonstruieren (Schimank 2009). Bestehende Lücken in dieser Rekonstruktion werden „synthetisierend“ mit der Wahl geeigneter „Werkzeuge“ zu füllen versucht. Die Beschäftigung mit soziologischen Rätseln kann „die Theoretikerin dazu anregen, sich nach geeigneten Werkzeugen umzuschauen, etwa in den speziellen Soziologien oder den Nachbardisziplinen, und notfalls selbst zur Werkzeugmacherin zu werden“" (Schimank 2016: 352).

Reckwitz hingegen lehnt sowohl den Ansatz einer funktionalen Differenzierung als auch einen akteurtheoretischen Ansatz, wie er z. B. von Schimank vertreten wird, ab (Reckwitz 2008c). Um die tradierte Fixiertheit der Differenzen zwischen den sozialen Funktionssystemen oder Klassen und den damit verknüpften Dualismus zu überwinden, plädiert Reckwitz für poststrukturalistische bzw. praxistheoretische Gegenpositionen, die „die Annahme einer Eindeutigkeit von sozialen Sinngrenzen für unrealistisch halten, und (die) vielmehr systematisch die chronischen Uneindeutigkeiten dieser Grenzen herausarbeiten, die [...] Hybridität der Kultur, in der Sinnelemente nicht separiert nebeneinander, sondern in komplexen, häufig 
auch immanent widersprüchlichen Kombinationen von Sinnelementen verschiedener Herkunft vorkommen" (Reckwitz 2008c: 4153).

In diesem Zusammenhang kann die von Reckwitz kritisierte synthetisierende und universalisierende Theorieentwicklung in der Soziologie, wie z. B. die akteurtheoretische Soziologie von Schimank, selbst als Beispiel für eine (Soziale) Innovation durch unintendiertes bzw. transintentionales Handeln interpretiert werden. Denn, so die Begründung von Reckwitz, es verhält sich so, „dass die Theorieentwicklung in der Soziologie die eigentümliche Form einer Generierung neuer theoretischer Differenzen gerade durch die fortwährende Formulierung von Synthesevokabularen genommen hat. Theoriesynthesen wollen das Theoriefeld ,schließen', tatsächlich und unintendiert aber öffnen sie dieses Feld für die Produktion von Gegenvokabularen." (Reckwitz 2007: 1)

Reckwitz distanziert sich von der wissenschaftstheoretischen Konzeption einer sukzessiven Annäherung an die Wahrheit bzw. einer sich kontinuierlich steigernden Problemlösefähigkeit, wie sie der Kritische Rationalismus vertritt. Er erkennt in den Universalisierungsversuchen vielmehr, wie eben beschrieben, „unintendierte Differenzgeneratoren“ (Reckwitz 2007), wobei sich die Diskurse als alternativlos darzustellen versuchen, „ihre eigene Kontingenz als Sinnsystem zu invisibilisieren, sich als die, natürliche Sicht der Dinge darzustellen, kurz: sie versuchen sich als ,universalen Horizont` zu installieren." (Reckwitz 2007: 3)

\subsection{Vergleich der Subjektkonzepte}

In diesem Abschnitt wird die Verwendungsweise des Subjektbegriffs durch einen Vertreter des akteurtheoretischen Ansatzes $\left(\mathrm{P}_{1}\right)$, repräsentiert durch die akteurtheoretische Soziologie von Uwe Schimank, dargestellt. Im nachfolgenden Kapitel wird die Verwendungsweise des Subjektbegriffs durch einen Vertreter der poststrukturalistischen Praxistheorie $\left(\mathrm{P}_{2}\right)$ gegenübergestellt.

2.1.1 Subjektkonzept des akteurtheoretischen Ansatzes von Schimank

Schimank vertritt die Position, dass über die determinierenden sozialen Strukturen hinaus eine Wahlfreiheit von Akteuren besteht, und warnt vor strukturalistischen Fehlschlüssen:

„Zumindest in einer [...] handlungstheoretischen

Perspektive sind Akteure keine bloßen Marionetten struk- tureller Zwänge. Akteure können sich diese Zwänge oft reflexiv vergegenwärtigen und sich ihnen auf dieser Basis dann prinzipiell auch entziehen." (Schimank 2013: 26)

Schimank lehnt sich bei der Interpretation der subjektiven Seite des Handelns, des Sinns und der Intentionalität von individuellem und sozialem Handeln stark an Max Weber und den Phänomenologen Alfred Schütz und Thomas Luckmann an (Schimank 2016: 28ff.). Handeln als subjektiv sinnhaftes Verhalten meint demnach bewusstes absichtsvolles Handeln, ein intentionales motivationales Tun. Selbst unbewusstes Tun, das ja im Alltag und im sozialen Zusammenleben sehr häufig auftritt, wird dabei als intentional aufgefasst, d. h. auch routiniertes Handeln hat nach dieser Theorie intentionalen Charakter.

Die Konstitution von Sinn erfolgt in einem bewussten Akt durch die „sukzessive Verdichtung des Bewusstseinsstromes zu Erlebnissen und der Verdichtung dieser Erlebnisse zu Erfahrungen [...]. Sinn wird als reflexive Relationierung von Erlebnissen mit anderen Erlebnissen konstituiert.“ (Schimank 2016: 31) Es besteht also ein Dualismus zwischen dem „Drinnen“ des Bewusstseins seiner selbst und seiner Körperlichkeit und dem „Draußen“ der wahrnehmbaren Geschehnisse der Welt, die immer wieder Anlass zu sinnhaften $\mathrm{Zu}$ - und Umordnungsvorgängen im bestehenden Erfahrungsschatz geben.

„Das Ergebnis der phänomenologischen Konstitutionsanalysen von Sinn lautet daher, dass Sinn auf einem innerbewusstseinsmäßigen Prozess der Selbstauslegung beruht und in Selbstverstehen besteht, und in dieser Form auch immer nur Sinn für ein Subjekt ist. "(Schimank 2016: 32)

Motivationales Handeln ist immer auf die Zukunft gerichtet. Über Zukunftsentwürfe wird der Ablauf des Tuns gesteuert. Schimank unterscheidet mit Alfred Schütz „Um-zu-Motive“ und „Weil-zu-Motive“, wobei die „Um-zu-Motive“ für das Handeln konstitutiv sind. In diesem Sinn liegt Handeln genau dann vor, „wenn jemand Ausschnitte seines erlebten Verhaltensstroms über zukunftsgewandte Um-zu-Motive selbst versteht“ (Schimank 2016:33). Um-zu-Motive sind immer bewusst. Weil-zu-Motive hingegen als ,subjektiver Ausdruck der sozialen Prägung des Handelns durch die ,Logik der Situation“" (Schimank 2016: 33) sind nicht unbedingt bewusst, wie z. B. unbewusstes Ausführen von Routinehandlungen. Handlungsentwürfe (Um-zu-Motive) entstehen nicht aus dem Nichts, sondern sie ergeben sich immer aus der Verwobenheit mit Vorgeschichten zurückliegender Erfahrungen (Weil-zu-Motive). 
In diesen Passagen, die Schimank in seinem Buch Handeln und Strukturen (2016) ausführt, kommt eine sozialphänomenologische Orientierung zum Ausdruck. Als wesentlich für das handelnde Subjekt lassen sich daraus folgende Merkmale ablesen:

- ein Dualismus zwischen subjektiver Konstituierung der Welt und einer objektiven Außenwelt, eine ontologische Innen-Außen-Differenz;

- eine durchgängige Intentionalität der sinnkonstitutiven und auf die Zukunft gerichteten kausalen Tätigkeit;

- eine souverän rationalistische Grunddisposition.

Aus praxeologischer bzw. poststrukturalistischer Sicht handelt es sich dabei um eine mentalistische Theorie, in der menschliches Verhalten als Produkt mental-intentionaler Akte und universeller Gesetze eines geistigen Inneren zum Ausdruck kommt (Moebius 2008: 6off.). Der Einfluss des cartesischen Dualismus scheint unverkennbar.

Zusammenfassend lässt sich sagen: Die Doppelperspektive des akteurtheoretischen Ansatzes versucht einerseits die Dichotomie zwischen Akteur und sozialer Struktur zu überwinden und beide Komponenten zu integrieren. Vorausgesetzt wird dabei aber dennoch die Subjektkonzeption eines rational intentionalen Dualismus zwischen Subjekt und Objekt, wenn auch nicht so radikal, wie es in der (neoklassischen) ökonomischen Theorie oder der Rational Choice Theory der Fall ist.

\subsubsection{Subjektkonzept der Praxistheorie von Reckwitz}

Kontrastierend zum akteurtheoretischen Modell des Subjekts, das im vorigen Abschnitt erläutert wurde, folgt in diesem Kapitel die Darstellung der praxeologischen Verwendung des Subjektbegriffs $\left(\mathrm{P}_{2}\right)$, repräsentiert durch die Praxistheorie von Andreas Reckwitz.

Reckwitz kritisiert das oben beschriebene (sozialphänomenologische) Subjektkonzept - wie auch den Mentalismus des Strukturalismus - als Variante eines Mentalismus in der Nachfolge des cartesischen Dualismus. Er sieht in diesem Dualismus und in der Ontologisierung des Geistes als einer eigenständigen Sphäre ein Grundproblem der intentionalen Handlungstheorien, insbesondere der beiden Paradigmen des Homo oeconomicus und des Homo sociologicus. In den praxeologischen Handlungstheorien wird demgegenüber „nicht von der Vorgegebenheit von subjektiven Interessen oder kollektiven Normensystemen“ (Reckwitz 2004: 320) ausgegangen. Handlungsmuster sind den kulturtheoretischen Ansätzen zufolge, wozu auch die Praxistheorien zu zählen sind, eingebettet in symbolische Wissensordnungen. Im Unterschied zu mentalistischen Kulturtheorien wird jedoch in den praxeologischen Ansätzen ,in verschiedener Weise der Versuch unternommen, den Zusammenhang zwischen Wissen und Verhalten jenseits des Musters einer Innen-Außen-Differenz zu denken“ (Reckwitz 2004: 320). Von entscheidender Bedeutung dabei ist nun, dass als Ort bzw. Einheit des Sozialen der sozialtheoretischen Überlegungen nicht mehr kognitive Vorgänge bzw. subjektive Konstruktionsbildungen verwendet werden, sondern die einzelnen sozialen Praktiken, wie z. B. Praktiken des Konsumierens, des Verhandelns, des Umgangs mit technischen Artefakten etc. Diese Praktiken haben den Charakter von Repetitionen (Reckwitz 2004).

„Der Begriff der ,Praktik' setzt [...] von vornherein an der Repetitivität eines Komplexes von Aktivitäten an, der ,typischerweise' von verschiedenen Individuen in der zeitlichen Sequenz zu unterschiedlichen Zeitpunkten und in verschiedenen räumlichen Settings hervorgebracht wird. " (Reckwitz 2004: 321)

Der Körper bzw. die körperliche Performance stellt nach der praxeologischen Perspektive kein Instrument dar, um ein Ziel zu erreichen. Es verhält sich vielmehr so:

„In sozialen Praktiken sind routinisierte Körperbewegungen und implizite, sequentiell eingesetzte Formen des Wissens und Verstehens zwei Seiten des gleichen Phänomens: [...] der Ort dieser kollektiven Wissens- und Verstehensformen [ist] nirgendwo sonst zu finden als in den ,öffentlichen'Verhaltensweisen: Wissensordnungen drücken sich damit in Bewegungsmustern aus. " (Reckwitz 2004: 322

Der in der Tradition der Handlungstheorien marginalisierte Körper, der hier als „Exekutor“ von Motiven und Normen oder kognitiven Schemata gesehen wurde, und die ebenfalls marginalisierte Repetitivität gleichförmigen Handelns rücken in der Praxistheorie ins Zentrum der Theorie.

„Die Theorien sozialer Praktiken thematisieren deutlich stärker als die klassischen Handlungstheorien die materialen Grundlagen des Handelns, vor allem die Körperlichkeit des Handelns aber auch die materiale Kultur der Artefakte, wie sie in die sozialen Praktiken integriert ist." (Reckwitz 2004: 325)

Das Subjekt ist in diesem Sinn weniger Denken als Tun und es nimmt Formen und Gestalten an, indem es an sozialen Praktiken partizipiert. Es ist in diesem 
Sinne einer kulturellen Ordnung bzw. einem kulturellen Code unterworfen. Körperliche und psychische Merkmale akzeptabler Subjekthaftigkeit werden dem Subjekt inkorporiert. Subjekte existieren nach dieser Theorie nur als bzw. im Vollzug von Praktiken, sie sind Produkte kulturspezifischer Praktiken.

Der praxeologische Ansatz impliziert eine spezifische Form von Innovationstheorie. Der innovative Generator besteht darin, dass den Repetitionen und deren Interpretationen das Potenzial permanenter Destabilisierung und Subversion inhärent ist: Aus zufälligen Abweichungen, interpretativen Mehrdeutigkeiten und impliziten Unsicherheiten, insbesondere in komplexen Konstellationen, entstehen neue Handlungsformen und neue soziale Praktiken als Ergebnis sozusagen misslungener praktischer Problemlösung im Sinn der Reproduktion stabiler Strukturen. Durchbrechungen und Verschiebungen von Routinen sind also immer möglich, und dies in einer kontingent und kulturhistorisch geprägten Welt, die universeller Strukturen hinter der Mannigfaltigkeit oder essentialistischer Fundierungen des Handelns entbehrt. Die Logik der Praxis besteht nicht nur aus absoluter Wiederholung von Routinen, sondern es ergibt „sich hier auch immer wieder eine interpretative und methodische Unbestimmtheit, Ungewissheit und Agonalität [...], die kontextspezifische Umdeutungen von Praktiken erfordert und eine ,Anwendung' erzwingt und ermöglicht, die in ihrer partiellen Innovativität mehr als reine Reproduktion darstellt“ (Reckwitz 2003: 294). Die Logik der Praxis, verstanden als Dynamik von Reproduktion/ Repetition und inhärentem Subversionspotenzial, erzwingt gleichsam permanent Veränderung und Innovation.

Im Gegensatz zu rationalistischen Handlungsmodellen ist der Akteur bzw. das Subjekt in der praxeologischen Perspektive durch Unkontrolliertheit und Unberechenbarkeit, damit aber auch durch kulturelle Innovation gekennzeichnet, „ohne dass dazu klassisch subjekttheoretisch eine Autonomie, Reflexivität oder Eigeninteressiertheit, des Subjekts' jenseits und vor der Praxis präjudiziert würde“ (Reckwitz 2003: 296). Innovation ergibt sich aus der Unberechenbarkeit in der „Überschneidung und Übereinanderschichtung verschiedener Wissensformen [...], in der praxeologischen Struktur des Subjekts als ein lose gekoppeltes Bündel von Wissensformen" (Reckwitz 2003: 296, Hervorh. im Original). Aus der Sicht der poststrukuralistischen Praxistheorie von Reckwitz scheitert zwar Widerspruchsfreiheit und Stabilität des Subjekts als kulturelles Gebilde an den unberechenbaren Überlagerungen und Inkohärenzen, profitiert aber sozusagen indirekt von dem sich daraus ergebenden Veränderungs- und Innovationspotenzial.

Reckwitz bezieht sich bei seiner Analyse der Unkontrollierbarkeit des Sinns in den diversen kulturellen Phänomenen vorrangig auf Jacques Derrida: „Ich möchte von vornherein eine spezifische Form poststrukturalistischen Denkens auswählen - jene Jacques Derridas - und erläutern, wie sie aus meiner Sicht helfen kann, gegen bestimmte Annahmen der sozialtheoretischen Tradition vertraute Phänomene in einer alternativen, verschobenen Perspektive zu betrachten." (Reckwitz 2008c: 4152)

Aufgehoben wird in der Praxistheorie nicht nur der Subjekt-Objekt-Dualismus, sondern auch die Dichotomie zwischen Mensch und Technik, insofern auch technischen Artefakten Handlungsfunktion zugeschrieben wird. Artefakte sind demnach nicht nur Teil von Handlungen, sondern selbst Träger von Handlungen, die unabhängig von Menschen mit dem Begriff Interobjektivität zusammengefasst werden. Einige Praktiken entstehen erst in diesem engen Zusammenhang mit Artefakten, sie wären ohne diese gar nicht möglich (Reckwitz 2003, 2004, 2016).

\subsection{Diskussion und Schlussfolgerungen}

Kontrastierend zum akteurtheoretischen Modell des Subjekts, das im vorigen Abschnitt erläutert wurde, folgt in diesem Kapitel die Darstellung der praxeologischen Verwendung des Subjektbegriffs ( $\left.\mathrm{P}_{2}\right)$, repräsentiert durch die Praxistheorie von Andreas Reckwitz.

Die zwei unterschiedlichen theoretischen Zugänge, der akteurtheoretische und der praxeologische, implizieren unterschiedliche Interpretationen und Verwendungsweisen des Begriffs der Intention und damit verbunden unterschiedliche Konzeptionen von Innovation. Die differente Bedeutung von Intention ergibt sich vor allem aus den unterschiedlichen Subjektkonzepten der beiden in Diskussion stehenden Theorien. Das klassische dualistisch rationale Subjektkonzept ist weniger dynamisch und bedarf eines Intentionsaspekts, um in Anpassung an eine sich permanent verändernde Welt und entsprechend den Bedürfnissen des Menschen notwendige Innovationen gezielt herbeizuführen. Die Intention scheint als notwendige konzeptionelle Brücke zu dienen, um die statische transsubjektive Struktur eines metaphysischen Dualismus von Innen und Außen in einen bewussten 
und steuerbaren lebensweltlichen Zusammenhang $\mathrm{zu}$ bringen.

Schimank übernimmt den klassischen rationalistischen Ansatz, differenziert diesen jedoch durch die Hinzunahme der Transintentionalität und die Veränderung der sozialen Einheit von einer reduktionistischen Akteur-Zentriertheit zu einem Konzept des Wechselspiels zwischen Akteur und Struktur. Die Handlungen der Akteure sind weiterhin als Grundelemente allen sozialen Geschehens anzusehen, die soziale Einheit ist aber im Sinn einer sozialen Dynamik als handelndes Zusammenwirken zu verstehen. Die Konstellationsstrukturen der Akteure sind „Beweger“ des Geschehens:

„Nicht einzelne Akteure jeweils für sich, sondern erst deren handelndes Zusammenwirken erzeugt, im Wechselspiel mit handlungsprägenden Strukturen, soziale Dynamiken." (Schimank 2009: 209)

Dieser Aspekt wird von systemtheoretischen Ansätzen komplett ausgeblendet, auf Akteure wird verzichtet, die soziale Einheit bilden in diesem Ansatz Kommunikationssysteme (Schimank 2009). Nach Schimank ergeben jedoch einzelne intentionale Handlungsimpulse einen „Summenvektor“ (handelndes Zusammenwirken), der von den einzelnen Vektoren weit abweichen kann, der aber in Richtung und Stärke nur aus diesen entstehen kann (Erscheinung der Transintentionalität). Es kann auch eine rein intentionale Strukturgestaltung auftreten, diese kann wiederum von transintentionalen Struktureffekten begleitet werden (Schimank 2016: 234f.)

Handeln wird aus praxeologischer Sicht nicht durch subjektive Motive, Intentionen oder Interessen erklärt, auch nicht durch Regel- und Normgeleitetheit, sondern über die sozialen Praktiken bzw. Performances als die Einheit des Sozialen. Handlungen sind in diese Praktiken eingebettet, in ihnen kommt als wissensbasierte Tätigkeit ein praktisches Wissen, ein praktisches Verständnis zum Einsatz, und ein „know how im Sinne eines impliziten Wissens, im Gegensatz zu „knowing that“ als explizierbarem Aussagewissen von Überzeugungen.

Der Begriff der Intention wird im Kontext einer Praxistheorie im Allgemeinen bzw. in der speziellen kulturtheoretischen Ausformung im Besonderen von Reckwitz stark relativiert. Intention im subjektiven Sinn ist nach dem praxeologischen Subjektkonzept gegeben, spielt aber bei der Entstehung von (sozialer) Innovation keine bedeutende Rolle. Der Begriff der Intention ist folglich nur bedingt als Abgrenzungskri- terium zwischen Sozialer Innovation und Sozialem Wandel anwendbar.

Die beiden dargestellten Ansätze, der akteurtheoretische und der praxeologische, erscheinen als sehr unterschiedliche und sich in großen Teilen widersprechende Paradigmen der Soziologie nicht kompatibel bzw. nicht ineinander überführbar. Im Hinblick auf die Fragestellung des Begriffs Intention als Abgrenzungskriterium ergibt sich, dass aus akteurtheoretischer Sicht Intentionen - so wie aus Sicht der klassischen handlungstheoretischen Ansätze - teilweise als Unterscheidungsmerkmal betrachtet werden können, insofern es sich um keine rein transintentionalen sozialen Geschehnisse handelt. Aus praxeologischer Perspektive ergibt es keinen Sinn, Soziale Innovationen von Sozialem Wandel anhand des Begriffs der Intention zu unterscheiden, da die soziale Einheit der Praktiken als überindividuelle Vorgänge konzipiert sind, obgleich subjektiv erlebte Intentionen keineswegs bestritten werden.

Aus der Vergleichsanalyse ergeben sich folgende Schlussfolgerungen in Bezug auf den Gebrauch des Begriffs Intention als Abgrenzungskriterium zwischen Sozialer Innovation und Sozialem Wandel:

Die Relativierung des Begriffs der Intention im Zusammenhang der praxeologischen Subjektkonstituierung einerseits und im Zusammenhang der Funktion eines Unterscheidungsmerkmals zwischen Sozialer Innovation und Sozialem Wandel andererseits könnte so interpretiert werden, dass die Auffassung Sozialer Innovation als Folge bzw. Potenzial fehlerhafter sozialer Repetitionen und Reproduktionen und der damit verbundenen Verschiebungen, Resignifizierungen und Subversionen als Erklärung für transintentionale Vorgänge angesehen werden könnte.

Wenn man sich (so wie Howaldt, Kopp und Schwarz in ihrer Schrift Zur Theorie sozialer Innovation. Tardes vernachlässigter Beitrag zur Entwicklung einer soziologischen Innovationstheorie unter Bezugnahme auf die von Gilles Deleuze wiederentdeckte intentionskritische Imitationstheorie von Gabriel Tarde Anfang des 20. Jahrhunderts) für die Präferenz eines praxistheoretischen Ansatzes ausspricht, dann erscheint eine gleichzeitige Bezugnahme auf eine handlungstheoretische Position der Intentionalität widersprüchlich und als Vermischung zweier Sprachen - einer subjektdezentrierten praxeologischen und einer akteur- und subjektzentrierten Sprache.

Basierend auf den Ergebnissen und Interpretationen dieser Vergleichsanalyse scheint es widersprüch- 
lich, wenn man sich aus dem Wunsch heraus, Soziale Innovation von technisch-ökonomischer Hegemonie $\mathrm{zu}$ emanzipieren, paradigmatisch auf die neuen praxeologischen Ansätze bezieht und zugleich weiterhin Intentionalität als wesentliches Unterscheidungsmerkmal zwischen Sozialer Innovation und Sozialem Wandel betont, wie es scheint, in Fortsetzung der Tradition von Wolfgang Zapf und Katrin Gillwald, die den Begriff, nach meiner Interpretation, innerhalb des rationalen handlungstheoretischen Paradigmas verwenden (Zapf 1994; Gillwald 2000).

Schon Tarde relativiert die Bedeutung der individuellen Intentionen bzw. die Interessengeleitetheit des Individuums im Hinblick auf die Generierung von Innovationen (Rothe 2012).

„For Tarde, innovation does not arise from the selfinterest of individual, but from the cooperation of many actors. Furthermore, Tarde emphasizes ,psychological forces, the role of passions and convictions - that is, the irrational, ,un-economic' aspects of the joint action of participants in the innovation process. In Tarde's view, destruction is not mere occupation of unknown territory, but the beginning of a collective, translation' in the course of which the innovation comes out." (Rothe 2012: 4)

Es drängt sich auch die Frage auf, ob das akteurtheoretische Paradigma, das an das Vokabular eines autonomen und souveränen Subjekts gebunden ist und selbst Züge eines technisch-ökonomischen Rationalismus in sich trägt, geeignet ist, Soziale Innovation von einer technisch-ökonomischen Dominanz zu emanzipieren. Konsequenterweise entwickelt Reckwitz aus seiner poststrukturalistisch inspirierten Praxistheorie heraus eine überhaupt neue Bedeutung von Innovation, die die Entwicklungen der Spätmoderne bzw. Hypermoderne widerspiegeln sollte, nämlich das Regime des kulturästhetisch Neuen.

„Während das Regime des Neuen der organisierten Moderne von der Eigendynamik technologischer Entwicklung [und daneben von politischen Reformbemühungen] angetrieben ist, ist das kulturell-ästhetische Regime des Neuen der Spätmoderne im Kern von den flottierenden Konsumentenwünschen, der Eigendynamik des Sektors der Konsumgüter und der Massenmedien, nicht zuletzt der digitalen Medien angetrieben. " (Reckwitz 2016: 266)

Der Vergleich des praxistheoretischen Ansatzes mit dem akteurtheoretischen Ansatz sollte zeigen, dass die Verwendung des Begriffes der Intention paradigmatisch auf sehr unterschiedliche und widersprüchliche Weise erfolgt. U. a. ergibt sich aus der Analyse die Notwendigkeit, die Abgrenzung zwischen bzw. das Verhältnis von Sozialer Innovation und Sozialem Wandel neu zu thematisieren und zu interpretieren.

\section{Das Dortmunder Forschungsprogramm}

Das Dortmunder Forschungsprogramm stellt den Versuch dar, die im vorangegangenen Kapitel besprochenen Paradigmen des akteurtheoretischen bzw. praxeologischen Ansatzes ( $\mathrm{P}_{1}$ und $\mathrm{P}_{2}$ ) innerhalb eines Forschungsprogramms zu kombinieren. Dieser Versuch der parallelen Anwendung zweier sich widersprechender Paradigmen mit dem Ziel, den Begriff der Sozialen Innovation theoretisch tiefergehend $\mathrm{zu}$ fundieren, wird im folgenden Abschnitt nachgezeichnet.

Das Dortmunder Forschungsprojekt um Howaldt, Jacobsen, Kopp, Schwarz et al. verfolgt das Ziel, Soziale Innovation durch eine (poststrukturalistische) Praxistheorie $\left(\mathrm{P}_{2}\right)$ theoretisch $\mathrm{zu}$ fundieren. Innerhalb dieses Forschungsprogramms kommt jedoch zugleich ein (traditionelles) akteurtheoretisch intentionales Paradigma ( $\left.\mathrm{P}_{1}\right)$ zur Anwendung. Die Frage der Vereinbarkeit dieser beiden Paradigmen innerhalb eines Forschungsansatzes wird im anschließenden vierten Kapitel auf logischer, inhaltlicher und wissenschaftstheoretischer Ebene kritisch hinterfragt.

Ausgehend von den neuen Herausforderungen der Globalisierung und Digitalisierung, des Übergangs von der Industrie- zur Wissens- und Dienstleistungsgesellschaft scheint ein Überdenken der technologisch-ökonomisch dominierten Konzeption des Innovationsbegriffs notwendig zu sein. Die Forderung nach einer Neubewertung von (Sozialer) Innovation kann als ethisch/moralischer Appell verstanden werden, denn nur ein neues Innovationsparadigma könne eine Verbesserung z. B. in der Sozialpolitik, Forschungspolitik, Arbeitspolitik, Gesellschaftspolitik oder Umweltpolitik herbeiführen (Howaldt 2014b).

„There is a growing consensus among practitioners, policy makers and the research community that widespread social innovation is required to cope with the significant challenges that societies are facing now and in the future." (Howaldt 2014b: 1)

Zugespitzt formuliert, hängt die nachhaltige Gestaltung der Zukunft der Gesellschaft von der Installation eines neuen Innovationsparadigmas bzw. einer adäquaten Innovationspolitik ab. Soziale Innovationen nehmen im gegenwärtigen gesellschaftlichen Kontext zu Unrecht einen sekundären Stellenwert hinter technisch-ökonomischen Innovationskonzepten ein (Howaldt et al. 2014a; Howaldt/Jacobsen 2010). Wün- 
schenswert wäre für diese Forscher*innen die Institutionalisierung des Konzepts der Sozialen Innovation im Sinn eines neuen Leitbildes.

Man könnte dies in Analogie zur Institutionalisierung der technologisch-ökonomisch definierten Innovation sehen.

„While invention had dominated modern concepts of the production of novelty, i.e. means of the establishment of the patent system and industrial research laboratories and their rapid expansion between 1920 and 1960, the term innovation inherited the meaning of invention. [...] this kind of institutionalization led to a situation where innovation was increasingly defined by and restricted to its technological meaning and its economic contribution."

(Knoblauch 2014: 10)

Damit Soziale Innovation als die dominante Form von Innovation institutionalisiert werden kann, ist aus Sicht der Dortmunder Forschungsgruppe jedoch eine theoretische Fundierung unumgänglich. Dies soll im Rahmen des Paradigmas einer poststrukturalistischen Praxistheorie $\left(\mathrm{P}_{2}\right)$ geschehen, wie im folgenden Kapitel 3.1 beschrieben. Parallel dazu wird jedoch das „traditionelle" intentional akteurtheoretische Paradigma ( $\left.\mathrm{P}_{1}\right)$ weiterhin aufrechterhalten, das im darauffolgenden Kapitel 3.2 zur Diskussion gestellt wird.

\subsection{Soziale Innovationen sind neue soziale Praktiken (Paradigma 2)}

\section{Theoretische Fundierung der Sozialen Innovation}

Soziale Innovation soll mehr sein „als nur Voraussetzung, Begleiterscheinung und Folge von technologischen Innovationen" (Howaldt/Jacobsen 2010: 97).

Aus der Beobachtung gegenwärtiger sozialer Problemlagen und der ethisch-normativen Forderung nach nachhaltigen Lösungen ergibt sich die Notwendigkeit, zuallererst das Konzept der Sozialen Innovation theoretisch zu fundieren, was bisher aus Sicht der Dortmunder Forschungsgruppe nur unzureichend und unsystematisch geschehen sei.

„Dies verweist auf und erfordert die Entwicklung einer bislang ausstehenden gesellschaftstheoretisch fundierten Konzeption sozialer Innovation als generativer Mechanismus sozialen Wandels." (Howaldt et al. 2017: 143)

Für die theoretische Fundierung der Sozialen Innovation sowie des Sozialen Wandels fällt die Wahl auf das Paradigma der Praxistheorie. Ein wichtiger Vorläufer des praxeologischen Ansatzes ist der wiederentdeckte Soziologe Gabriel Tarde. Dieser Klassiker der Soziologie, der seine Hauptwerke Ende des 19. und Anfang des 20. Jahrhunderts verfasste, erfuhr in den letzten Jahren eine wahre Renaissance in unterschiedlichsten Wissenschaftsdisziplinen (Howaldt 2014a). Lange Zeit wurde dieser „Poststructuralism before Structuralism“ (Moebius 2004: 18) durch die Soziologie Émile Durkheims überschattet. Doch seit dem sogenannten Practice Turn in den Neunzigerjahren wurde die „Nachahmungstheorie“ Tardes mit großem Interesse rezipiert und er wurde als zentrale Figur der entstehenden Praxistheorie gefeiert. Den Status eines bedeutenden Wegbereiters einer praxeologisch orientierten Sozialtheorie nimmt Tarde auch im Dortmunder Forschungsprojekt ein (Howaldt et al. 2014a). Tarde wird aber nicht nur als Vordenker einer sozialen Praxistheorie gesehen, sondern auch als Theoretiker, der viele wesentliche Aspekte des Poststrukturalismus vorweggenommen hat.

Es gibt jedoch unterschiedliche Versionen von Praxistheorien, die sich alle mehr oder weniger noch in einem Entwicklungsstadium befinden. Es handelt sich um ein work in progress, das aus verschiedenen, heuristisch mehr oder weniger fruchtbaren Ansätzen heraus erst operationalisierbare Hypothesen und Theorien formulieren muss. Thematisch nicht näher eingegangen wird in den Arbeiten der Dortmunder Autor*innen auf den performativen Aspekt und die Implikationen des praxeologischen Innovationsparadigmas auf die Konzeption des Subjekts (Howaldt/Jacobsen 2010; Howaldt 2014a; Howaldt 2014b; Howaldt et al. 2017).

Durchgängig poststrukturalistisch/praxeologisch (P2) konzipiert ist die Auffassung von Sozialer Innovation als Etablierung neuer sozialer Praktiken als differentielle Wiederholung, wie in den nachfolgenden Passagen dargestellt.

Soziale Innovation als differentielle Wiederholung

Das in der Performativität eingelassene innovative Potenzial entspricht dem Konzept der Nachahmung, das in sich die permanente Möglichkeit der Abweichung aufweist. In poststrukturalistischer Lesart der Praxistheorie und in Übereinstimmung mit Tarde konstituiert sich das Subjekt durch performative Akte. In der Wiederholung ist in diesem Sinn die Erfindung enthalten, denn in jeder Wiederholung steckt ein Mindestmaß an Veränderung und Variation, die sich gerade durch die Repetitionen in einem Möglichkeitsraum realisieren und manifestieren. Grundlegend ist das von Tarde vorweggenommene Prinzip der Iterabilität, eine permanente performative Iteration, die durch 
ihre Zitierfähigkeit und Fehlerbehaftetheit innovative Effekte erzeugt.

"Iterability allows a sign to evade from it's context, to confuse and to go beyond its context and to repeat itself in another context; in this connection the sign can be quoted differently or, in a wrong way', it can multiply and yet obtain another meaning. " (Moebius 2004: 20)

Die Erfindung zielt auf die Nachahmung und die Nachahmung auf die Variation. In teleologischer Diktion könnte man sagen, die nachahmende Wiederholung existiert um der Variation willen. Die (metaphysische) Ausgangssituation wird dabei von Tarde als eine umfassende Heterogenität konzipiert, die sich über Erfindung und Nachahmung zu homogenisieren und $\mathrm{zu}$ stabilisieren sucht.

„Neben dieser immer schon vorausgesetzten Heterogenität wird aber auch die eigentlich homogenisierende Wiederholung zur Schöpferin von Erfindungen." (Borch/ Stäheli 2009: 16)

In der Nachahmung ist immer auch Variation enthalten. Den Nachahmungsprozessen sind Neuerungsprozesse inhärent, die zu innovativen sozialen Praktiken diffundieren können (Howaldt et al. 2014a: 34ff.). Der Ort der Innovation wird weg vom Subjekt und weg vom Objekt auf die Praktiken selbst verlagert. Es sind nicht postulierte Eigenschaften eines autonomen Subjekts, die Veränderungen der Praktiken bewirken, sondern Eigenschaften der sozialen Praktiken selbst, es ist die "Logik der Praxis“ (Bourdieu 1972), der ein dynamisches Innovationspotenzial innewohnt (Reckwitz 2003, 294ff.).

Im Kapitel 3.2 folgt die Darstellung der akteurtheoretischen Komponente des Forschungsansatzes, in der ein autonom und intentional agierendes Subjekt $\left(\mathrm{P}_{1}\right)$ für die Definition von Sozialer Innovation veranschlagt wird.

3.2 Diskussion des akteurtheoretischen Subjekt- und Intentionsbegriffs im Rahmen des Dortmunder Forschungsprogramms (Paradigma 1)

Die folgende Diskussion der Anwendung des akteurtheoretischen Subjekt- und Intentionsbegriffs im Dortmunder Forschungsprogramm soll auch das Schwanken zwischen den beiden Paradigmen veranschaulichen.

Die von Tarde beschriebenen und analysierten Nachahmungsprozesse ereignen sich nach Moebius' Interpretation $\mathrm{zu}$ überwiegendem Maße nicht intentional, großteils unbewusst und quasi automatisch (Moebius 2009).
"Society is imitation and imitation is a kind of somnambulism ... Consequently, to be credulous and docile, and to be so as pre-eminently as the somnambulist and the social man, is to be, primarily, imitative. To innovate, to discover, to awake for an instant from his dream of home and country, the individual must escape, for the time being, from his social surroundings. Such unusual audacity makes him super-social rather than social." (Tarde 1962: 87, zit. in Moebius 2004: 19)

Nach dem Ansatz der Dortmunder Soziolog*innen „sind Erfindung und die Nachahmung der dahinterstehenden Intentionalität die untrennbar miteinander verwobenen Treiber des Wandels“ (Howaldt et al. 2014: 39). Unter Bezugnahme auf Moebius kann dieser Prozess als ,iterative Dynamisierung von sozialen Strukturen“ aufgefasst werden (Moebius 2009).

Die Einfügung des Ausdrucks „dahinterstehende Intentionalität" scheint jedoch problematisch, denn Moebius stellt eindeutig fest, dass

„repetition is a social logic; social structures generate and exist because of repetition; these processes are not effects of the will of individuals but are unconscious, unintended and beyond rational choices" (Moebius 2004: 17).

Wenn auch von Moebius eingeräumt wird, dass es auch bewusste Nachahmungsprozesse gibt, schreibt er doch unter Bezugnahme auf Tarde und die poststrukturalistische Denkrichtung (vorrangig Jacques Derrida und Judith Butler) den unbewussten Prozessen eine weitaus größere Bedeutung zu, wenn er diese sogar als „quasi-automatic processes“ beschreibt (Moebius 2004: 19). Eine interessante Frage ist jedoch, auf welche Weise dieser "social somnambulism“ (Moebius 2004: 2o) unterbrochen wird.

"The social renewal, transformation or invention however is like a meta-social (sur-or super-social) awakening from this hypnotic sleep in a tiny moment." (Moebius 2004: 20)

Ereignen sich in diesen „tiny moments“ im Strom der Repetition subjektive und bewusste Aneignungsprozesse, in denen in einem performativen Akt aus Wahrgenommenem Neues erzeugt und erlebbar gemacht wird? Wenn man von der These ausgeht, dass nicht nur die Menschen die Objekte beeinflussen, sondern auch die Objekte als quasi handelnde Akteure auch die Menschen, kann man den Prozess der Aneignung als Verflechtung sozialer Praktiken und Objekte, die in ihrer Materialität gleich bleiben, aber das Denken der Menschen verändern, interpretieren. Was sich in diesem Aneignungsprozess verändert, ist die Relation zum und der strukturierte Umgang mit dem Objekt, 
indem der Kontext des Objekts, des Neuen und Fremden, modifiziert wird.

Nach Howaldt und Jacobsen werden die Routinen unterbrochen, „wenn eine Situation für die Handelnden durch Anomalien beziehungsweise neue Erfahrungen problematisch wird und sie dadurch zu einer reflexiven Einstellung gegenüber der Situation zwingt" (Howaldt/Jacobsen 2010: 103).

Einerseits hat es den Anschein, Innovationsprozesse würden im Sinn einer Komplexitätstheorie aufgefasst: Emergente Prozesse als Eigenschaft komplexer Systeme sind „unintendierbar", ereignen sich zufällig, sind kausal nicht rekonstruierbar, sind nicht vorhersehbar und in starkem Maße kontextabhängig.

Dies widerspricht andererseits dem Anspruch, dass das neue Innovationsprogramm, das die „zunehmende Bedeutung sozialer Innovationen als eigenständige Innovationsform“ (Howaldt et al. 2017: 145) widerspiegeln soll, institutionell arrangierte, gesellschaftliche Selbststeuerungs- und Gestaltungsprozesse (Howaldt et al. 2017) übernehmen könnte. Denn Soziale Innovationen im Sinne Tardes und der Praxistheorien sind kontingent und unkontrollierbar, und daher nicht steuerbar. Nachahmungsprozesse verlaufen vielmehr zum großen Teil unbewusst und unintendiert.

„The processes of repeating are not an expression of conscious individuals who decide rationally but are essentially processes that carry on unconsciously, unintended and beyond purely rational calculations." (Moebius 2004: 27)

Die Dortmunder Forschungsgruppe konzediert die Kontingenz und den Indeterminismus des mikrosoziologischen Ansatzes Tardes bzw. der poststrukturalistischen Praxeologie und gesteht zu, dass es sich „um nicht-teleologische hochgradig kontingente Prozesse handelt" (Howaldt et al. 2014: 38), die nicht intendiert sein können, da sich nur intendieren lässt, was auch als konkretes Ziel erwartbar ist.

„Vor diesem Hintergrund wird auch deutlich, dass soziale Innovationen sowohl in ihrer konkreten Erscheinungsform als auch in ihrem Entstehungsprozess im eigentlichen Sinne gar nicht intendiert sein können." (Howaldt et al. 2014: 38)

Auch die Interpretation von Christian Borch und Urs Stäheli (Borch/Stäheli 2009) geht in die Richtung, dass der Nachahmungsprozess zwar von Individuen getragen wird, ,aber diese handeln in der Regel nicht bewusst" (Borch/Stäheli 2009: 10).

„Mag aus einer handlungstheoretischen Sicht die

Frage nach der bewußten Intention von Handlungen von
Bedeutung sein, so interessiert diese Frage Tarde so wenig, daß sie nur einen Nebenschauplatz in seiner Theoriebildung darstellt: Manche Nachahmungen mögen bewußt zustande kommen, viele andere nicht. Die Differenz ist letztlich grundbegrifflich bedeutungslos, da Nachahmungen sich nicht durch die subjektiven Intentionen einzelner erklären lassen." (Borch/Stäheli 2009: 10)

Soziale Praktiken können nach Tarde demnach nicht als vektorielle Summe intentionaler Handlungen betrachtet werden, und wenn die Nachahmung als soziologisches Gesetz gelten soll, so wie von Howaldt, Kopp und Schwarz postuliert, scheint dies nicht kohärent mit dem Ziel zu sein, eine intentional gedachte Steuerung und Gestaltung gesellschaftlicher Vorgänge durch eine sozialwissenschaftlich bekehrte Innovationspolitik zu installieren, und auch nicht mit dem Versuch, einen erwünschten Sozialen Wandel in Gang zu setzen, indem man Soziale Innovationen als Treiber des Sozialen Wandels bewusst entwickelt, durchsetzt und verbreitet (Howaldt et al. 2014a: 91-95).

Sowohl der strukturalistische als auch der akteurtheoretische Mentalismus enthält unreflektierte (nicht dekonstruierte) Voraussetzungen, die als universelle Eigenschaften aufgefasst werden. Kollektiv-mentales Wissen, kollektive Wissensordnungen gelten als Bedingung oder Ursache von kollektiven Handlungsmustern, es wird damit eine „,innere Sphäre` mentaler Gegebenheiten präjudiziert, die scheinbar unabhängig von sozial-kulturellen Attributen existieren" (Reckwitz 2000: 184). Die Welt wird dichotomisiert in Subjekt und Objekt, wobei das Subjekt, die innere Sphäre, äußerliches Verhalten und Handlungen bestimmt und intentional steuert. Sowohl das individuell Mentale als auch das kollektiv Mentale wird ontologisiert bzw. essentialisiert, ist aber nach poststrukturalistischer Auffassung nur ein Konstrukt von vielen möglichen. Dieses dualistische Subjektkonzept wird von den Vertreter ${ }^{\star}$ innen der Praxistheorie stark bezweifelt und kritisiert, stattdessen wird das soziale Geschehen in der Performativität sozialer Praktiken verortet (Reckwitz 2000).

„Mentale Eigenschaften existieren in der Sozialwelt nicht , an sich, sondern dadurch, dass sie sich in Körperbewegungen, im alltäglichen Tun - und dies schließt auch Sprechakte ein - ,ausdrücken." (Reckwitz 200o: 177)

Diesen Mechanismus eines performativen Innovationsgenerators gilt es gemäß Dortmunder Forschungsprogramm intentional und normativ bzw. innovationspolitisch im Sinn eines akteurtheoretischen Paradigmas zu steuern. Darin zeigt sich der Wider- 
spruch: Das Subjekt der poststrukturalistischen Praxistheorie ist ein dezentriertes, ein $\mathrm{zu}$ dekonstruierendes, keine mentalistisch intentionale Entität. Darin sind sich die unterschiedlichen Versionen der Praxistheorie einig, dass eine derartige Ontologisierung des Subjekts $\mathrm{zu}$ verwerfen ist. Die Intentionalität wird sozusagen vom aktionstheoretischen Subjekt bzw. strukturalistischen Objekt in die Praktiken hineinverlegt.

Aus meiner Sicht werden damit zwei paradigmatisch unterschiedliche Ansätze zum Einsatz gebracht, ein praxeologischer mit einem sich dekonstruierenden dezentrierten Subjekt und ein akteurtheoretischer mit einem intentional agierenden Subjekt. Zu klären wäre, inwieweit aus einer theoretischen Inkohärenz heraus der normativ ethische Anspruch einer Verbesserung der Gesellschaft zu realisieren ist.

Im abschließenden Teil der Arbeit wird die parallele Anwendung der sich widersprechenden akteurund praxistheoretischen Paradigmen innerhalb des Dortmunder Forschungsprogramms zur Sozialen Innovation auf logischer, inhaltlicher und wissenschaftstheoretischer Ebene kritisiert.

\section{Kritik der parallelen Anwendung von akteur- und praxistheoretischem Paradigma in der Erforschung von Sozialer Innovation}

Der Widerspruch der parallelen Anwendung von akteur- und praxistheoretischem Paradigma innerhalb eines Forschungsprogramms zur sozialtheoretischen Untersuchung von Sozialer Innovation bildet sich auf logischer, inhaltlicher und wissenschaftstheoretischer Ebene ab. Dabei ist anzumerken, dass rationales Argumentieren auf einer (wissenschaftstheoretischen) Metaebene vorausgesetzt wird, auch wenn, wie in der poststrukturalistischen Praxistheorie, gerade die Irrationalität und Unkontrollierbarkeit des menschlichen Handelns hervorgehoben wird. D. h. es müssen Widersprüche zugelassen werden (tertium non datur) - das Postulat zum Beispiel einer Komplexität bzw. Polykontexturalität des Sozialen ohne intentional agierendes Subjekt widerspricht einem akteurzentrierten Ansatz mit intentional agierenden autonomen Subjekten.

i) Die Kritik auf logischer Ebene besteht darin, dass aus zwei sich widersprechenden Axiomen bzw. Satzsystemen alles und nichts gefolgert werden kann - kompakt zusammengefasst in dem lateinischen Satz „Ex contradictione sequitur quodlibet“, formal ausgedrückt: $(\mathrm{A} \wedge \neg \mathrm{A}) \rightarrow \mathrm{B}$. Sofern man diesen Satz der klassischen Logik akzeptiert, besteht der Sachverhalt, dass aus zwei sich widersprechenden Aussagen jeder beliebige Satz gefolgert werden kann, unabhängig von Personen und verwendeten Inhalten.

Die parallele Anwendung von sich widersprechenden bzw. stark divergierenden Paradigmen ( $\mathrm{P}_{1}$ und $\mathrm{P}_{2}$ ) zur Erklärung von Prozessen der Sozialen Innovation würde dementsprechend zu Fehlschlüssen führen bzw. nicht rechtfertigbare Schlussfolgerungen erlauben. Eine paradigmatische Vermischung, die vielleicht für einen postmodernen bzw. postfaktischen Relativismus kein Problem darstellen mag, blockiert jedoch meiner Ansicht nach die Forschungsperspektiven, die sich im Rahmen einer (poststrukturalistischen) Praxeologisierung von Sozialer Innovation eröffnen.

ii) Nach dem praxistheoretischen Ansatz ereignen sich Innovationen in der Ausübung der Praxishandlung selbst. Das akteurtheoretische Konzept intentional agierender Subjekte, das das Dortmunder Forschungsprogramm zusätzlich in das neue Innovationsparadigma integrieren will, steht, wie in Kapitel 2 erörtert, im Widerspruch zu theoretischen Kernannahmen der Praxistheorie, insbesondere zur Version der poststrukturalistischen Praxistheorie, wo innovative Vorgänge prinzipiell kontingent, emergent, indeterministisch und weitgehend unvorhersehbar verlaufen.

Wie Howaldt und Schwarz selbst formulieren, verändern die „zahl- und namenlosen Erfindungen und Entdeckungen [...] durch ebenso zahllose Nachahmungshandlungen die Gesellschaft und ihre Praktiken und werden erst durch diese zu einem wahrhaft sozialen Phänomen" (Howaldt/Schwarz 2014a: 34). Und diese zahllosen Nachahmungshandlungen sind unbewusst und unintendiert. Schon Tarde wendet sich gegen das Konzept eines freien Willens und „betont die Eingebettetheit jeglicher Erfindung in ein dichtes Netz von Nachahmungsströmen“" (Howaldt/Schwarz 2014a: 34, Anmerkung 15). Sozialer Wandel bzw. Soziale Innovation ist aus praxistheoretischer Sicht ein „kontingentes Phänomen [...], das sich jeder allgemeinen (Makro-) Theorie widersetzt, weil in jeder reproduzierenden Handlung und in der prinzipiellen Aushandlungsbedingtheit sozialer Strukturen zugleich die Möglichkeit der Veränderung und damit ein riesiges Feld möglicher Transformationen steckt" (Howaldt/Schwarz 2014a: 37).

In der Praxis treten eine Vielzahl von unbeabsichtigten Folgen und Konsequenzen Sozialer Innovationen in Erscheinung, die akteurtheoretische Ansätze in argumentative Verlegenheit bringen: unintendierte Effekte (transintentionale Prozesse), unerwünschte 
Nebeneffekte (wie z. B. Rebound-Effekte, Backfire, Transformations-Effekte, Widerstands-Effekte), gegenteilige Effekte, Ambivalenz in der Bewertung, Wechsel des Referenzrahmens mit gleichzeitigem Übergang intendierter Handlungen in transintentionale Vorgänge bzw. Interferenzen von Referenzen in einer komplexen Innovationsgesellschaft (Rammert 2013).

Selbst wenn man intentional agierende und steuernde Subjekte einräumt, so ist eine angestrebte und realisierte Neuerung, insbesondere in einer komplexen Gesellschaft, unterschiedlichsten Kontexten ausgesetzt, die die Innovationen auf unvorhersehbare Weise auch wieder verändern. Bruno Latour betont vor allem auch die Veränderung der Resultate von intendierten Innovationen durch die Objekte selbst, die ähnliche Effekte wie die Akteure bewirken können:

„Wer hat je einen Konstrukteur gesehen, der seine Konstruktion beherrschte? Wo ist der Schöpfer, der sich fähig fühlte, seine Schöpfung zu kontrollieren? Welcher Erfinder von Robotern dünkt sich ihr Gebieter, welchem Marionettenspieler haben seine Marionetten nicht überraschende Kunststücke beigebracht?" (Latour 2016: 202)

Das Forschungsprogramm einer praxeologischen Fundierung des Begriffs der Sozialen Innovation, wie es die Dortmunder Forschungsgruppe vorschlägt, scheint ein heuristisch fruchtbarer und vielversprechender Ansatz zu sein, der aus meiner Sicht zu neuen Einsichten in die Entstehungszusammenhänge und Relationen von Sozialem Wandel und Sozialer Innovation beiträgt. $\mathrm{Zu}$ kritisieren ist allerdings die paradigmatische Parallelführung praxeologischer und akteurtheoretischer Ansätze. Ein Rückgriff auf das Vokabular der Moderne ist nicht nur logisch unhaltbar, sondern scheint auch inhaltlich blockierend und verzerrend zu wirken.

iii) Beim Theorienvergleich in Kapitel 2 wurde auch eine divergierende wissenschaftstheoretische Orientierung deutlich. Während akteurtheoretische Ansätze bzw. die Rational-Choice-Theorie ein deduktiv-nomologisches Modell favorisieren, begleitet von dem Bedürfnis, die unbefriedigende Fragmentierung in einen einheitlichen Ansatz zu überführen, treten Praxeologen und Poststrukturalisten offen für einen Theorienpluralismus ein, in dem vor allem Entstehungszusammenhänge, weniger Begründungszusammenhänge, fokussiert werden. Eine Popper'sche Approximation an die Wahrheit wird verworfen, es geht vielmehr um „die Anerkennung einer irreduziblen Pluriperspektivität auf das Soziale“ (Schäfer 2016: 25).

Auf der Seite der Praxistheorie besteht offenbar ein entgegengesetztes Interesse nach Multiperspektivität:
„Es kann daher nicht um die Reduktion, sondern nur um eine Entfaltung von Perspektiven gehen." (Schäfer 2013: 25) Inakzeptabel erscheint aus dieser Sicht ein falsifikationistischer Ansatz im Sinne des Kritischen Rationalismus, begrüßenswert die Konzeption von vielfältigen Perspektiven innerhalb eines praxeologischen Paradigmas, die aufgrund der Komplexität des Sozialen auch widersprüchliche Beschreibungen und Paradoxien aufweisen können (Schäfer 2013: 25ff.).

Als Beispiel einer dekonstruktiven Wissenschaftstheorie sei die exzellente Untersuchung zum experimentaltheoretisch entdeckten genetischen Codierungssystems in der Proteinbiosynthese von Hans-Jörg Rheinberger angeführt. Die Dekonstruktion Rheinbergers zeigt ein eigendynamisches System eines Netzwerks von Praktiken, „das von Laboratorien, Instrumente und Experimentalarrangements strukturiert wird“ (Rheinberger 2006: 232). Artefakte, Kontingenz, Emergenz, Hybridisierung von Experimentalsystemen bilden die Bestandteile der „Denkmaschine“ zur Erzeugung von Ereignissen, die über die Absichten der Experimentator*innen hinausgeht und zu unvorhersehbaren Wandlungen des „epistemischen Objekts“ führt.

„Man könnte sagen, das, was im nachhinein den

Beteiligten zunehmend als Intention erschien, bildete sich im Laufe ihres Forschungsprozesses als Raum möglicher Intuition erst heraus. " (Rheinberger 2006: 268)

Es handelt sich um eine Experimentalstrategie ohne Finalität, eine Strategie nicht zielgerichteter Bewegungen, die in ihren Wiederholungen und Rekontextualisierungen Differenzen und Verschiebungen erzeugt. Rheinberger interpretiert erfolgreiches wissenschaftliche Experimentieren als „différance“ im Sinne Derridas. Die bloße Anwendung von Methoden, in der kontingente Abweichungen unmöglich wären bzw. in der man Abweichungen verhindern wollte, würde nichts Neues entstehen lassen, würde sozusagen einen Widerstand gegen Innovation bedeuten.

„Experimentalsysteme, die so angelegt sind, daß die Proliferation von Differenzen zum Orientierungsprinzip ihres eigenen Fortwirkens wird, sind durchdrungen von und schaffen gleichzeitig jenen subversiven Impetus, den Jacques Derrida ,différance' genannt hat. Dieser Begriff zielt auf eine, wie Derrida sich ausdrückt, ,irreduzible Abwesenheit der Intention, die aller Erfindung eigentümlich ist, und schärft damit die Aufmerksamkeit für die ,Ereignishaftigkeit', die Forschungspraxis auszeichnet." (Rheinberger 2006: 99; Derrida 1976: 151)

Auch in der experimentellen Naturwissenschaft werden nach Rheinberger Entdeckungen und Innova- 
tionen über Fehler in der Wiederholung und Reproduktion auf nicht vorhersehbare Weise generiert. Bifurkation und Hybridisierung zwischen Experimentalsystemen bilden eine „Maschine zur Erzeugung von Ereignissen. [...] Die dynamische Masse des Wissens, das Netzwerk von Praktiken, das durch Laboratorien, Instrumente und Experimentalarrangements strukturiert wird, ist eine Denkmaschine sui generis.“ (Rheinberger 2006: 232) Analog zu den Thesen der Praxistheorie in den Sozial- und Kulturwissenschaften entsteht Neues in Experimentalsystemen der Biologie aus einem Wechselspiel von Stabilität und Instabilität sowie einem Prozess differentieller Wiederholung. Aus einem In-vitro-System der Proteinbiosynthese ist z. B. die Transfer-RNA nicht durch demiurgische Eingriffe genialer Wissenschaftler oder zielgerichteter Interventionen hervorgegangen, sondern: „Das Molekül wurde gefunden, ohne daß danach gesucht worden war. Und ausgerechnet dieses Molekül erwies sich als Brücke, über die sich die Proteinsyntheseforschung mit der Molekularbiologie verknüpfen ließ." (Rheinberger 2006: 17)

Wenn man Experimentalsysteme, egal ob naturwissenschaftliche oder sozialwissenschaftliche, offen für eine differentielle Reproduktion hält, dann werden nach der Logik der Iterabilität Überraschungen und Neuerungen über Verschiebungen und Rekontextualisierungen generiert. Die intentionale Anwendung von Methoden reproduziert etablierte Strukturen, führt zu keinen Erfindungen (Rheinberger 2006: 97ff.).

„Letztlich ist jede Innovation in einem grundlegenden Sinn ein Resultat - vielleicht eher noch ein Zufall, ein Abfall - solcher Reproduktion." (Rheinberger 2006: 90)

Sozialer Wandel wäre aus dieser Sicht nichts anderes als dieser Strom performativer Iterabilität in einem Netzwerk sozialer Praktiken, die relational verschoben, subversiv umgestaltet, resignifiziert und rekontextualisiert werden können. Soziale Innovationen könnten in diesem Rahmen als die Handlungen definiert werden, die bei Störungen eines praxeologischen Gleichgewichts über mikrologische Aneignungs- und Transformationsprozesse stabilisierend gegensteuern.

Urs Stäheli weist auf die Affinität von Poststrukturalismus und Erkenntnissen der Komplexitätstheorie hin (Stäheli 2008). Abschließend wird diese spannende Forschungsperspektive für eine praxeologische Interpretation von Sozialer Innovation (ohne Rückgriff auf ein akteurtheoretisches Paradigma) in einem Ausblick kurz umrissen.

\section{Forschungsperspektive: Praxeologischer Ansatz und Komplexitätstheorie in Bezug auf Soziale Innovation}

Der Ansatz der poststrukturalistischen Praxistheorie scheint nicht kompatibel mit einem akteurtheoretischen, weist jedoch viele Ähnlichkeiten mit Ansätzen aus der Komplexitätstheorie bzw. einer neuen Systemtheorie auf (Stäheli 2008). Ein Thinking in Complexity erscheint vielen Autor ${ }^{\star}$ innen als Notwendigkeit, um Herausforderungen wie die Bildung transnationaler Systeme, dezentrierter Demokratien oder Migrationsgesellschaften im Kontext von Globalisierung und Digitalisierung zu bewältigen (Mainzer 2004; Pantzar/ Shove 2010; Willke 2016).

„Erst wenn der mündige und rationale Mensch versteht, dass die gesellschaftlichen Verhältnisse - festgelegt in den Strukturen, Prozessen und Regeln des Systems nicht nur die komplementäre Seite möglicher individueller Autonomie sind (dies war immer schon Bestandteil eines aufgeklärten Begriffs von Freiheit), sondern sich in den verdeckten und tiefgründigen emergenten Eigenschaften manifestieren, kann er die Macht der Verhältnisse begreifen und lernen, mit ihnen umzugehen." (Willke 2016: 190)

Die praxeologische Annahme einer Dynamik von vernetzten Praktiken, die sozusagen eine eigene Welt neben Individuen und sozialen Strukturen ausbildet, befindet sich jenseits der Konzeption eines schumpeterianischen „phallogozentristischen“ Unternehmergenies - um einen Ausdruck von Judith Butler zu verwenden. Komplexe Systeme bilden Bifurkationen aus, Momente der Unentscheidbarkeit, die allein rational, im Sinn von kausal bzw. kausalintentional, nicht steuerbar und beherrschbar sind. Diese diachrone Innovationsdynamik komplexer sozialer Systeme (Sozialer Wandel) wird durchbrochen von synchroner spontaner Musterbildung (soziale, technische, ökonomische Innovationen), von „Musterwechsel in Ungleichgewichtssituationen“, mehr oder weniger unabhängig von Eigenschaften von Akteuren und Agency (Stäheli 2008: 120ff.).

In der Terminologie einer neuen Systemtheorie würde der hier beschriebene Soziale Wandel mit einer diachronen Emergenz von Neuem und die Soziale Innovation mit einer synchronen emergenten Musterbildung korrespondieren. Auch hier wird die Dichotomie zwischen Handlung und Struktur überwunden. Die eigenständige Dimension der (unbewussten) Praktiken entspricht Handlung und Struktur übergreifender selbstreferentieller Systeme, die in ihrer Dynamik 
unvorhersehbar, kausal nicht rekonstruierbar Neues generieren bzw. emergieren. - Als ethisches Korrektiv für eine derartige Konzeption von Innovation bietet sich im Anschluss an Levinas, Derrida und Butler die „Ethik des Anderen“ an, in Ablöse der utilitaristischen Ethik von Rational Choice und Neoliberalismus wäre eine alteritätsethische Perspektive von Sozialer Innovation zu entwickeln.

\section{Literatur}

Alkemeyer, Th./Bröckling U. (2018): Jenseits des Individuums. Zur Subjektivierung kollektiver Subjekte. Ein Forschungsprogramm. In: Alkemeyer, Th./Bröckling, U./Peter, T. (Hg.): Jenseits der Person. Zur Subjektivierung von Kollektiven. Bielefeld: transcript Verlag, 17-31. Online: https://www.researchgate.net/publication/323919002_ Alkemeyer_Brockling_Peter_Eds_2018_-_Jenseits_ der_Person_Zur_Subjektivierung_von_Kollektiven [05.11.2019]

Alkemeyer, Th./Buschmann, N./Michaeler, M. (2015): Kritik der Praxis. Plädoyer für eine subjektivierungstheoretische Erweiterung der Praxistheorien. In: Alkemeyer Th./Schürmann, V./Volbers, J. (Hg.): Praxis denken: Konzepte und Kritik. Wiesbaden: Springer, 26-50. Online: https://www.academia.edu/27104512/Kritik_der_Praxis [05.11.2019].

Bhabha, H. K. (200o): Die Verortung der Kultur. Tübingen: Stauffenburg Verlag.

Borch, Ch./Stäheli, U. (2009): Einleitung - Tardes Soziologie der Nachahmung und des Begehrens. In: Borch, Ch./ Stäheli, U. (Hg.): Soziologie der Nachahmung und des Begehrens. Materialien zu Gabriel Tarde. Frankfurt am Main: Suhrkamp Verlag, 7-38.

Bourdieu, P. (1972): Entwurf einer Theorie der Praxis. Frankfurt am Main: Suhrkamp Verlag.

Butler, J. (2007): Kritik der ethischen Gewalt. Frankfurt am Main: Suhrkamp Verlag.

Derrida, J. (1976): Signatur Ereignis Kontext. In: Derrida, J.: Randgänge der Philosophie. Frankfurt am Main: Ullstein Verlag, 124-155.

Esfeld, M. (2002): Was besagt semantischer Holismus? In Bertram, G. W./Liptow, J. (Hg.): Holismus in der Philosophie. Ein zentrales Motiv der Gegenwartsphilosophie. Weilerswist: Velbrück Wissenschaft, 41-58. Online: https://www.unil.ch/files/live/sites/philo/files/shared/ DocsPerso/EsfeldMichael/2002/Bertram-Liptow02.pdf [30.01.2020].

Foucault, M. (1974): Die Ordnung der Dinge. Frankfurt am Main: Suhrkamp Verlag.

Gabriel, M./Gratzl, N./Gruber, D. (2014): Zwischen akteurzentrierter und systemtheoretischer Soziologie. In: Kornmesser, St./Schurz, G. (Hg.): Die multiparadigma- tische Struktur der Wissenschaften. Wiesbaden: Springer, 305-335.

Gillwald, K. (2000): Konzepte sozialer Innovation. WZB Discussion Paper, No. P oo-519, Wissenschaftszentrum Berlin für Sozialforschung (WZB), Berlin. Online: https:// www.econstor.eu/bitstream/10419/50299/1/319103064. $\operatorname{pdf}[30.07 .2019]$.

Howaldt, J./Kopp, R./Schwarz, M. (2017): Experimentelle Praktiken und nachahmende Wiederholung: Überlegungen zu einer Theorie sozialer Innovationen. In: Böschen, St./Groß, M./Krohn, W. (Hg.): Experimentelle Gesellschaft. Das Experiment als wissensgesellschaftliches Dispositiv. Online: http://www.ciando.com/img/books/ extract/3845285451_lp.pdf [30.06.2019].

Howaldt, J./Kopp, R./Schwarz, M. (2014): Zur Theorie sozialer Innovation. Tardes vernachlässigter Beitrag zur Entwicklung einer soziologischen Innovationstheorie. Weinheim und Basel: Beltz Juventa.

Howaldt, J./Kesselring, A./Kopp, R./Schwarz, M. (2014): Social Theory. In: Howaldt, J./Butzin, A./Domanski, D./ Kaletka, C. (2014): Theoretical Approaches to Social Innovation - A Critical Literature Review. A deliverable of the project: „Social Innovation: Driving Force of Social Change" (SI-DRIVE). Dortmund: Sozialforschungsstelle, 9-20. Online: http://www.si-drive.eu/wp-content/ uploads/2014/11/D1_1-Critical-Literature-Review.pdf [30.06.2019].

Howaldt, J./Jacobsen, H. (2010): Soziale Innovation. Auf dem Weg zu einem postindustriellen Innovationsparadigma. Wiesbaden: VS Verlag für Sozialwissenschaften.

Howaldt, J./Schwarz, M. (2010): Soziale Innovation im Fokus. Jürgen Howaldt und Michael Schwarz im Interview mit Jürgen Schultze. Online: http://www.sfs.tu-dortmund. $\mathrm{de} / \mathrm{cms} / \mathrm{Medienpool} /$ small_publications/Interview_Soziale_Innovation_im_Fokus.pdf [15.03.2019].

Knoblauch, H. (2014): Communicative Action, Reflexivity, and Innovation Society. TUTS-WP, (3-2014) 1-18.

Kropp, C. (2017): Forschung zu sozialen Innovationen am Scheideweg. Reaktion auf zwei Beiträge zum Thema Soziale Innovationen in GAIA 26/3 (2017): Howaldt, J./Schwarz, M./Jaeger-Erben, M. et al. GAIA, 26 (4), 309-312. Online: https://www.researchgate.net/publication/321970616_Forschung_zu_sozialen_Innovationen_ am_Scheideweg [13.10.2019].

Latour, B. (2016): Jubilieren. Über religiöse Rede. Berlin: Suhrkamp Verlag.

Lemke, M./Schaal, G. S. (2014): Paradigmenpluralität in der Politikwissenschaft. In: Kornmesser, St./Schurz, G. (Hg.): Die multiparadigmatische Struktur der Wissenschaften. Wiesbaden: Springer, 63-101.

Mainzer, K. (2004): Thinking in Complexity. Berlin, Heidelberg: Springer.

Mayntz, R./Scharpf, F.W. (1995): Der Ansatz des akteurzentrierten Institutionalismus. In: Mayntz, R./Scharpf, F.W. (Hg.): Gesellschaftliche Selbstregulierung und politische Steuerung. Frankfurt am Main: Campus, 39-72. Online: https://www.uzh.ch/cmsssl/suz/dam/jcr:ffffffff- 
9ac6-46e7-00oo-00005763c3co/05.06_mayntz_95.pdf [15.10.2019].

Moebius, St. (2004): Imitation, Repetition and Iterability. Poststructuralism and the ,social laws' of Gabriel Tarde. Distinktion: Journal of Social Theory, 5 (2), 55-69. Online: https://www.researchgate.net/publication/233141101_ IMITATION_REPETITION_AND_ITERABILITY [15.08.2019].

Moebius, St. (2008): Handlung und Praxis: Konturen einer poststrukturalistischen Praxistheorie. In: Moebius, St./ Reckwitz, A. (Hg.): Poststrukturalistische Sozialwissenschaften. Frankfurt am Main: Suhrkamp Verlag, 58-74.

Moebius, St. (2009): Imitation, differentielle Wiederholung und Iterabiltät. In: Borch, Chr./Stäheli, U. (Hg.): Soziologie der Nachahmung und des Begehrens. Frankfurt: Suhrkamp Verlag.

Pantzar, M./Shove E. (2010): Temporal Rhythms as Outcomes of Social Practices. Ethnologia Europaea. Journal of European Ethnology, 40 (1), 2-18. Online: https://courses. helsinki.fi/sites/default/files/course-material/4682436/ pantzar_shove_2010.pdf [10.02.2020].

Rammert, W. (2013): Vielfalt der Innovation und gesellschaftlicher Zusammenhalt. Von der ökonomischen zur gesellschaftstheoretischen Perspektive. TUTS-WP-1-2013, 1-16. Online: https://www.researchgate.net/publication/298213423_Vielfalt_der_Innovation_und_gesellschaftlicher_Zusammenhalt_Von_der_okonomischen_ zur_gesellschaftlichen_Perspektive [10.06.2019].

Reckwitz, A. (200o): Der Status des ,Mentalen' in den kulturtheoretischen Handlungserklärungen. Zeitschrift für Soziologie, 29 (3), 167-185. Online: https://www.degruyter. com/downloadpdf/journals/zfsoz/29/3/article-p167.xml [12.06.2019].

Reckwitz, A. (2002): Toward a Theory of Social Practices: A Development in Culturalist Theorizing. European Journal of Social Theory, 5 (2), 243-263. Online: https://www. semanticscholar.org/paper/Theorizing-Toward-a-Theory-of-Social-Practices-\%3A-A-Reckwitz/2119do31d5ofbb 432f8bo2384485f7b2boc92bo6 [20.07.2019].

Reckwitz, A. (2003): Grundelemente einer Theorie sozialer Praktiken: Eine sozialtheoretische Perspektive. Zeitschrift für Soziologie, 32 (4), 282-301. Online: https:// www.praxisphilosophie.de/reckwitz_praktiken_sozialtheorie.pdf/ [10.06.2019].

Reckwitz, A. (2004): Die Entwicklung des Vokabulars der Handlungstheorien: Von den zweck- und normorientierten Modellen zu den Kultur- und Praxistheorien. In: Gabriel, M. (Hg.): Paradigmen der akteurszentrierten Soziologie. Wiesbaden: VS Verlag für Sozialwissenschaften, 303-328. Online: https://www.kuwi.europa-uni.de/de/ lehrstuhl/vs/kulsoz/professurinhaber/buecher_artikel/ reckwitz_2004a_.pdf [05.05.2019].

Reckwitz, A. (2007): Warum die ,Einheit' der Soziologie unmöglich ist: die Dynamik theoretischer Differenzproduktion und die Selbsttransformation der Moderne. In: Schimank, U./Greshoff, R. (Hg.): Was erklärt die Soziologie? Methodologien, Modelle, Perspektiven. Münster: Lit-
Verlag, 65-77. Online: https://www.kuwi.europa-uni.de/ de/lehrstuhl/vs/kulsoz/professurinhaber/buecher_artikel/reckwitz_2005c_.pdf [15.07.2019].

Reckwitz, A. (2008a): Subjekt/Identität: Die Produktion und die Subversion des Individuums. In: Moebius, St./Reckwitz, A. (Hg.): Poststrukturalistische Sozialwissenschaften. Frankfurt am Main: Suhrkamp Verlag, 75-92.

Reckwitz, A. (2008b): Moderne: Der Kampf um die Öffnung und Schließung von Kontingenzen. In: Moebius, St./ Reckwitz, A. (Hg.): Poststrukturalistische Sozialwissenschaften. Frankfurt am Main: Suhrkamp Verlag, 226244 .

Reckwitz, A. (2008c): Das „Drinnen im Draußen“ Analyseperspektiven poststrukturalistischer Sozialwissenschaft. In: Rehberg, K.-S. (Hg.): Die Natur der Gesellschaft. Teilbd. 1 u. 2. Frankfurt am Main: Campus Verlag, 4152-4157. Online: https://nbn-resolving.org/urn:nbn:de:0168ssoar-155070 [10.05.2019].

Reckwitz, A. (2016): Kreativität und soziale Praxis. Studien zur Sozial- und Gesellschaftstheorie. Bielefeld: transcript Verlag.

Rheinberger, H.-J. (2006): Experimentalsysteme und epistemische Dinge. Frankfurt am Main: Suhrkamp Verlag.

Rothe, K. (2012): Creative destructions: Gabriel Tarde's concept of a passionate economy. In: Lagaay, A./Lorber, M. (Hg.): Destruction in the Performative. Special Issue of Cultural Critque. Amsterdam, New York: Editions Rodopi, 179-194. Online: http://rothespraxis.de/wp-content/ uploads/2014/02/Rothe-TARDE2012.pdf [20.07.2019].

Schäfer, H. (2016): Praxis als Wiederholung. Das Denken der Iterabilität und seine Konsequenzen für die Methodologie praxeologischer Forschung. In: Schäfer, H. (Hg.): Praxistheorie. Ein soziologisches Forschungsprogramm. Bielefeld: transcript Verlag, 137-159. Online: https:// www.researchgate.net/publication/308141415_Praxis_ als_Wiederholung_Das_Denken_der_Iterabilitat_und_ seine_Konsequenzen_fur_die_Methodologie_praxeologischer_Forschung [01.11.2019].

Schimank, U./Werle, R. (2000): Gesellschaftliche Komplexität und kollektive Handlungsfähigkeit. In: Schimank, U./Werle, R. (Hg.): Gesellschaftliche Komplexität und kollektive Handlungsfähigkeit. Frankfurt, New York: Campus, 9-22. Online: https://www.econstor.eu/obitstream/10419/69273/1/735645736.pdf [01.05.2019].

Schimank, U. (2009): Wie sich funktionale Differenzierung reproduziert - eine akteurtheoretische Erklärung. In: Hill, P. /Kalter, F./Kopp, J./Kroneberg, C./Schnell (Hg.): Hartmut Essers Erklärende Soziologie. Kontroversen und Perspektiven. Frankfurt am Main: Campus. Online: https://www.uzh.ch/cmsssl/suz/dam/jcr:ffffffff-9ac6-46e7ffff-ffff83a435d9/05.27_schimank_09.pdf [30.07.2019].

Schimank, U. (2013): Ausgangspunkte. In: Schimank, U. (2013): Gesellschaft. Bielefeld: transcript Verlag, bes. 9-36.

Schimank, U. (2016): Handeln und Strukturen. Einführung in die akteurtheoretische Soziologie. 5. Auflage. Weinheim und Basel: Beltz Juventa. 
Stäheli, U. (2008): System: Unentscheidbarkeit und Differenz. In: Moebius, St./Reckwitz, A. (Hg.): Poststrukturalistische Sozialwissenschaften. Frankfurt am Main: Suhrkamp Verlag, 108-123.

Willke, H. (2016): Dezentrierte Demokratie. Berlin: Suhrkamp Verlag.

Zapf, W. (1994): Über soziale Innovationen. In: Zapf, W.: Modernisierung, Wohlfahrtsentwicklung und Transformation: soziologische Aufsätze 1987 bis 1994. Berlin, Edition Sigma, 23-40. Online: https://www.econstor.eu/bitstream/10419/122762/1/209671.pdf [30.07.2019]. 